2. P. M. Cohn, Unique factorization in noncommutative power series rings, Proc. Cambridge Philos. Soc 58 (1962), 452-464.

3. Y. Kawada, Cohomology of group extensions, J. Fac. Sci. Univ. Tokyo Sect. I 9 (1963), 417-431.

4. J.-P. Serre, Cohomologie Galoisienne, Lecture Notes in Mathematics No. 5, Springer, Berlin, 1964.

5. —- Corps locaux et isogénies, Séminaire Bourbaki, 1958/1959, Éxposé 185

6. - Corps locaux, Hermann, Paris, 1962.

UNIVERSITY OF MichigAN

\title{
COHOMOLOGY OF DIHEDRAL GROUPS OF ORDER $2 p$
}

\section{BY PHYLLIS GRAHAM}

Communicated by G. Whaples, November 29, 1965

Let $D$ be a dihedral group of order $2 p$, where $p$ is an odd prime. $D$ is generated by the elements $\alpha$ and $\beta$ with the relations $\alpha^{p}=\beta^{2}=1$ and $\beta \alpha \beta=\alpha^{-1}$. Let $A$ be the subgroup of $D$ generated by $\alpha$, and let $A_{0}, A_{1}, \cdots, A_{p-1}$ be the subgroups generated by $\beta, \alpha \beta, \cdots, \alpha^{p-1} \beta$, respectively. Let $M$ be any $D$-module. Then the cohomology groups $H^{n}\left(A_{0}, M\right)$ and $H^{n}\left(A_{i}, M\right), i=1,2, \cdots, p-1$ are isomorphic for every integer $n$, so the eight groups $H^{-1}(D, M), H^{0}(D, M), H^{1}(D, M)$, $H^{2}(D, M), H^{-1}(A, M), H^{0}(A, M), H^{-1}\left(A_{0}, M\right)$, and $H^{0}\left(A_{0}, M\right)$ determine all cohomology groups of $M$ with respect to $D$ and to all of its subgroups. We have found what values this array takes on as $M$ runs through all finitely generated $D$-modules.

All possibilities for the first four members of this array are determined as a special case of the results of Yang [4]. But we have not been able to extend his methods so as to determine all possibilities for the whole array; our methods are independent to those of Yang.

Method of Proof. First we follow the method of Parr [3] in showing that it suffices to consider only finitely generated $\tilde{Z}$-torsion free $\tilde{Z} D$-modules, where $\tilde{Z}$ is the ring of all fractions $m / n$ of rational integers $m$ and $n$ such that $(n, 2 p)=1$. Lee [2] has listed all indecomposable modules of this type; there are ten. We compute directly several of the cohomology groups for the first five modules in her list. The last five of her list may be treated as members of extensions. These yield exact sequences of cohomology groups, which give information about the last five modules. We can then complete the values in all of the arrays by using the result that the cohomology of 
a dimension shift for a finitely generated module $M$ must be a finite direct sum of cohomologies arising from the list of ten.

Results. If $M$ is any finitely generated $D$-module, then the cohomology of $M$ with respect to $D$ and to all its subgroups is given by the direct sum of finitely many of the following:

$\begin{array}{rcccccccc} & H^{-1} & H^{0} & H^{1} & H^{2} & H^{-1} & H^{0} & H^{-1} & H^{0} \\ & (D, M) & (D, M) & (D, M) & (D, M) & (A, M) & (A, M) & \left(A_{0}, M\right) & \left(A_{0}, M\right) \\ \text { 1. } & 0 & Z_{2 p} & 0 & Z_{2} & 0 & Z_{p} & 0 & Z_{2} \\ \text { 2. } & Z_{2} & 0 & Z_{2} & Z_{p} & 0 & Z_{p} & Z_{2} & 0 \\ \text { 3. } & 0 & Z_{p} & 0 & Z_{p} & 0 & 2 Z_{p} & 0 & 0 \\ \text { 4. } & Z_{p} & 0 & 0 & 0 & Z_{p} & 0 & 0 & 0 \\ \text { 5. } & 0 & 0 & Z_{p} & 0 & Z_{p} & 0 & 0 & 0 \\ \text { 6. } & 0 & Z_{2} & 0 & Z_{2} & 0 & 0 & 0 & Z_{2} \\ \text { 7. } & Z_{2} & 0 & Z_{2} & 0 & 0 & 0 & Z_{2} & 0 \\ \text { 8. } & 0 & Z_{p} & 0 & 0 & 0 & Z_{p} & 0 & 0 \\ \text { 9. } & 0 & 0 & 0 & Z_{p} & 0 & Z_{p} & 0 & 0 \\ \text { 10. } & 0 & 0 & 0 & 0 & 0 & 0 & 0 & 0\end{array}$

\section{REFERENCES}

1. H. Cartan and S. Eilenberg, Homological algebra, Princeton Mathematical Series 19, Princeton Univ. Press, Princeton, N. J., 1956.

2. M. Lee, Integral representations of dihedral groups of order $2 p$, Tech. Rep., Univ. of Illinois, Chicago, Ill., 1962.

3. J. Parr, Cohomology of cyclic groups of prime square order, Bull. Amer. Math. Soc. 70 (1964), 427-428.

4. K.-W. Yang, On some finite groups and their cohomology, Pacific J. Math. 14 (1964), 735-740.

INDIANA StATE UnIVERSITY 